\title{
A NEW APPROACH FOR AN INTELLIGENT SWARM ROBOTIC
} SYSTEM

\author{
Rajath U Joshi ${ }^{1}$, Shashank T $S^{2}$ \\ ${ }^{1}$ Student, Dept of Electronics, Dayananda Sagar College of Engineering, Karnataka,India \\ ${ }^{2}$ Student, Dept of Electronics, Dayananda Sagar College of Engineering, Karnataka,India
}

\begin{abstract}
Swarm robotics generally faces problems such as regular human interference, monitoring and lack of precision in accomplishing a task. These problems can be avoided by endorsing a new multi robot system. The tasks which are highly demanding and generally which cannot be accomplished by a single robot can be accomplished through Swarm Robotics which involves a group of robots. The multi robot system comprises of a drone(quadcopter) controlling and commanding the ground robots in order to accomplish the task. This approach preserves the versatility of each individual in the swarm and also establishes coordinative and cognitive behavior. The drone activates the ground robots and also monitors the movement of each ground robot. The ground robots also interact with each other to complete a task.This approach ensures complete autonomy and precision.
\end{abstract}

Key Words: multi robot, drone, autonomy, versatility, Swarm robotics.

\section{INRODUCTION}

Swarm behavior is generally exhibited by ants when there arises a need to defend their territories, when a relatively big chunk of food is to be moved so that the entire colony of ants can feed on it. The swarm behavior helps to accomplish a task which cannot be done by an individual. This approach involves a multirobot system comprising of a drone(quadcopter) and 5 ground robots. The drone is the control unit of the system. The drone activates the ground robots based on the requirement of the task and also monitors the movements of the ground robots. Each ground robot is versatile, that is it can perform a task which requires contribution from a single robot such as lifting a small cube and the ground robot can also accomplish tasks as a group like pushing a heavy object. The ground robots interact with each other through IR communication. In this approach each ground robot is lit with different colored LEDS and the camera mounted on the Drone detects the ground robots through color object detection and tracking .The drone is equipped with a camera connected to Raspberry Pi. If the task is to pick up a heavy object which certainly can't be accomplished by a single ground robot. Through Image Processing the distance between ground robots and the object is estimated and this value is transmitted to and received by the ground robots through $2.4 \mathrm{Ghz}$ transceiver. The robots which are activated by the drone cover the distance and then perform the task of lifting the object. This intelligent system requires no human supervision. This ground robots are completely autonomous and also adapt to the environment . The ground robots have the capability to arrange themselves based on priority and the ground robots also avoid collision through Proximity sensors on board.The system can be classified into two
i) Drone
ii) Ground Robots

\section{DRONE (QUADCOPTER)}

Drone is the control unit of the system . The drone is built using 4 brushless motors, electronic speed controllers(ESC)s ,they are used to vary the speed of brushless motors. The Pulse width Modulation(PWM) signals are given from microcontroller in order to vary the speed of the brushless motors. The MSP430 microcontroller is used . A MPU6050 6 axis (Accelerometer + Gyroscope) module is used to maintain the stability of the drone and maneuverability of the drone .Based on the feedback from MPU6050 the PWM signals are sent by the MSP430 to vary the speed of motors . The MPU6050 comes in handy to determine the Roll, Pitch and Yaw variations and also offset can be compensated based on the feedback from MPU6050 which measures the tilt and motion variations. The drone is equipped with a camera connected to Raspberry $\mathrm{Pi}$ in order to carry out image processing and through that color object tracking. The drone can be controlled through $2.4 \mathrm{GHz}$ transceiver. The drone can be made stick to the ceiling or roof so that the camera captures the entire arena comprising of ground robots and the object which has to be shifted .

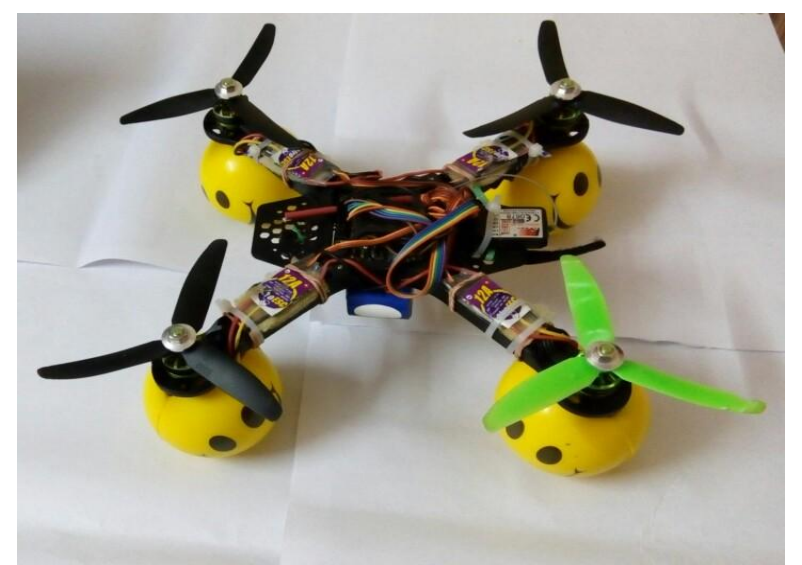

Fig-1: Figure depicts the drone(quadcopter). 


\subsection{COLOR OBJECT TRACKING}

The 5 ground robots are divided into 2 groups and an individual robot. There are 2 groups comprising of 2 robots each and a single robot.The robots in a group are lit up with with LED strips of same color.Both the robots in a group are lit up with LED strips of same color. If robots of one group are lit up with Red LED strips then the robots of the other group are lit up with Blue LED strips. 2 robots in each group are lit up with same color LEDs. The individual robot will have a color different from the rest. The Raspberry Pi $\mathrm{B}+(700 \mathrm{Mhz}$ Broadcom processor, 512MB RAM ,40 GPIO pins) is used for Image Processing task and the code to carry out Color object detection and tracking through Image Processing is written in Python using OpenCV library.The Color object tracking and detection is carried out based on the camera feed. First each frame is captured as an image from the video. The captured image is then converted to HSV(Hue,Saturation,Value) which is representation in cylindrical coordinate system. Then Image Thresholding is done to isolate colors and threshold values are set for each color. The Moments( weighted average of image Pixel intensities) are determined and then the $\mathrm{x}$ and $\mathrm{y}$ coordinates of the center of the object is determined by dividing the moments of each robot or object by its area.This is done by using a special OpenCV function called "GetSpatialMoment". Once the center is determined, in this case a common center of 2 robots in a group as the robots are lit up with same color and they are in close proximity it appears to the camera as a single entity. In the same way center of the object or the obstacle which has to be shifted is determined and through Euclid's Distance Formula the distance between the centers of robots and object is calculated. The ground robots are activated based on color. The ground robots can be activated based on color priority that is, if red colored robots are to be activated first ,then green and yellow at last . For this a priority of colors has to be given.The drone activates the group after detection of all the colors and based on the code written for priority of colors the group of ground robots are activated. The activation happens through $2.4 \mathrm{Ghz}$ transmitter and each ground robot has a $2.4 \mathrm{Ghz}$ receiver to receive the signal transmitted by the drone. The drone transmits a 2 digit Binary number through $2.4 \mathrm{Ghz}$ transmitter such as NRF24L01 and this signal is received by all the ground robots since all the robots are equipped with $2.4 \mathrm{Ghz}$ NRF24L01 receiver. The ground robots compare the 2 digit binary value with their assigned value, if the values match then those robots of a group get activated. The ground robots of the same group will have the same 2 digit binary value assigned . But robots belonging to different groups will be assigned different value.

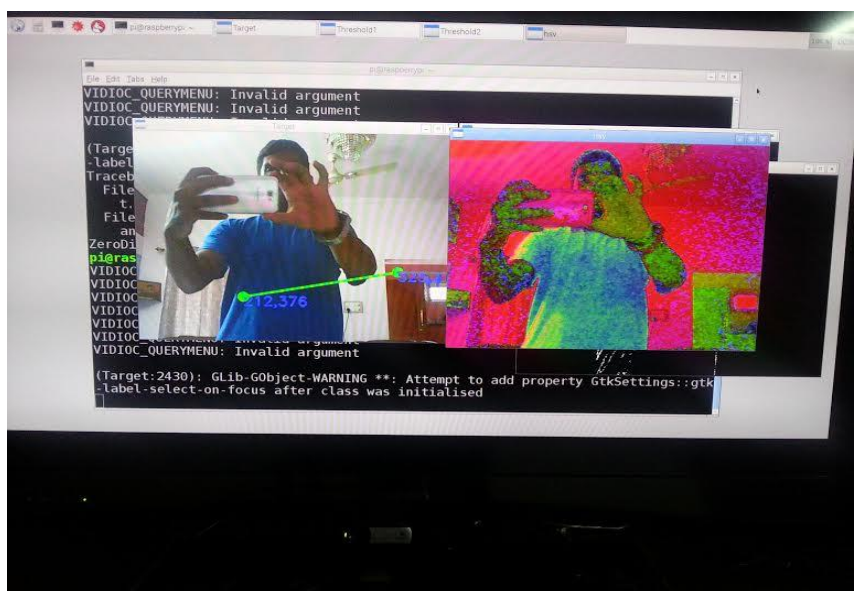

Fig-2: Window on the right showing HSV representation.

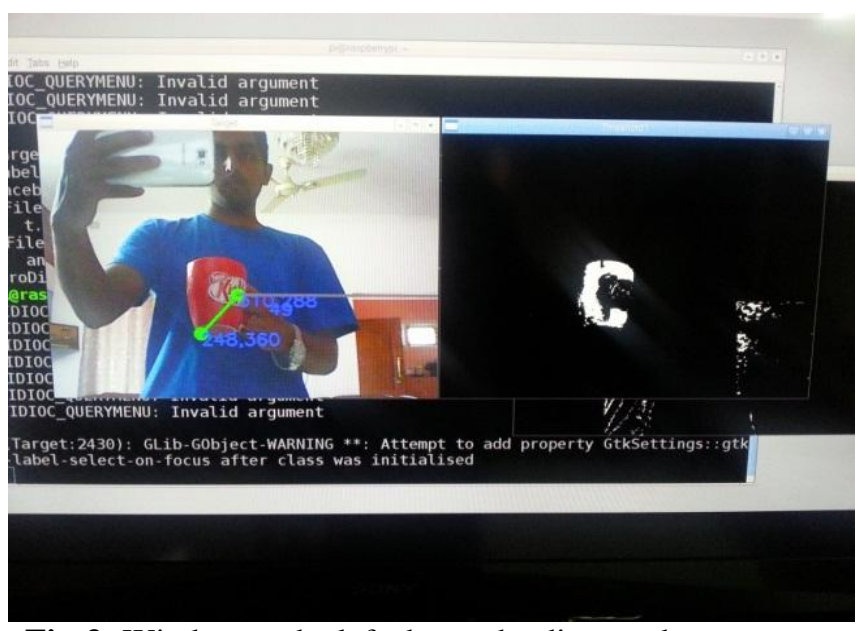

Fig-3: Window to the left shows the distance between two two objects of different color, in this case Blue and Red.

\section{Ground Robots}

Once a group gets activated, the drone transmits the distance between the group and the object or obstacle which has to be shifted. The first group which has been activated by the drone starts moving towards the object and starts covering the distance. Each ground robot is equipped with Ultrasonic Sensor(HC-SR04) in order to get a sense of distance. The ground robots cover the distance and approach the target that is the object with the help of ultrasonic sensor which measures the distance between the object and the robot. The ground robots are also equipped with HMC5883L(Digital Compass) which provides a sense of direction. The ground robots comprise of MSP430 microcontroller. The robots also have motors which are driven by 4 Channel Relay . 2 robots maintain a close proximity so that the camera sees the 2 robots lit up with same color as a single entity. This approach helps to determine the coordinate of center easily. If a robot deviates from its course or changes direction , based on the feedback from Digital Compass the microcontroller rectifies the offset and brings it back on course .Once the first group covers the distance the LEDS on the 2 robots turn off and detecting this the drone activates The next group the ground robots of that group travel the distance and then the LEDs on the robots belonging to second group also turn off. Detecting this the drone finally 
activates the last robot. The last robot also travels the same distance, the movements of robots will be tracked continuously by the drone. Once the LEDs on the last robot turn off, the drone detects no color and at this instance the drone sends a signal to all the ground robots. Once this signal is received by all the ground robots, the particular code concerned with shifting the object starts getting executed. Shifting involves picking up the object by all the 5 ground robots and placing it at a desired distance. All the ground robots begin the process of lifting the object simultaneously and also accomplish the task in unison. The ground robots are equipped with servo motor connected to a Tool or a structure which facilitates lifting. Each robot is equipped with IR sensor or Proximity sensor to prevent collision between one another. The ground robots communicate with each other through IR communication.

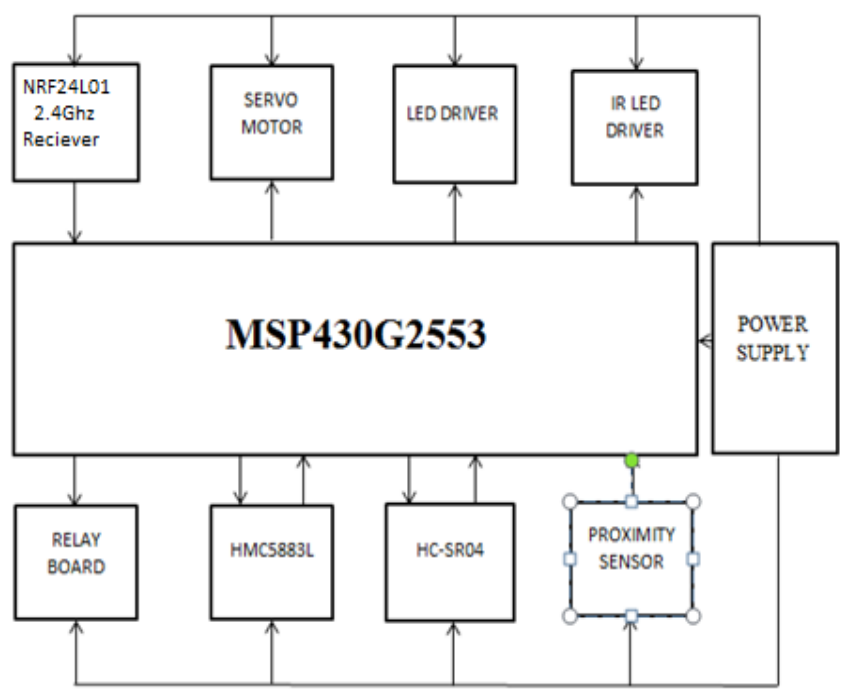

Fig-4:Block diagram of a Ground Robot.

\subsection{Automatic Priority based orientation}

The ground robots have the capability to autonomously arrange themselves based on priority. Each ground robot is assigned a number between 1 to 5 .The ground robots continuously transmit and receive the numbers through IR communication. Consider 2 robots. In a pair ,If the first robot is assigned a number 4 and second robot a number 3 , then the first robot after receiving the number of second robot compares it with its own number. Here the number received is 3 and its own number is 4 so the first robot if it is not situated behind the second robot starts to make way for the second robot so that the second robot supersedes the first robot of the pair. Similarly all the five robots align themselves based on priority. This process is facilitated by Ultrasonic Sensor, Digital compass, IR sensor or proximity sensor. Priority based arrangement is of great use. It will be helpful in accomplishing many tasks. A robot having a lower number than the other robot will always try to supersede the robot with higher value or number. Similarly the robots compare with each other and move upward or downward based on the assigned value. The robots either supersede or precede. The comparison takes place among all the robots until the desired arrangement is attained.

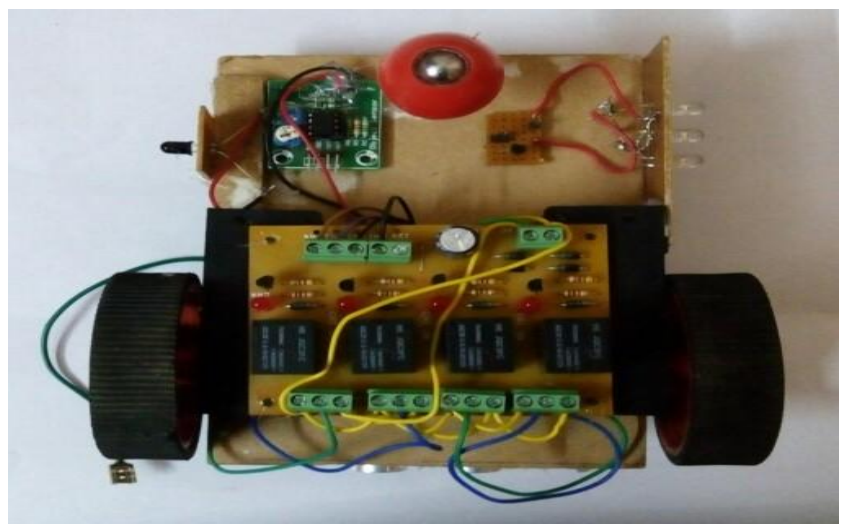

Fig-5: Bottom view of a ground robot.

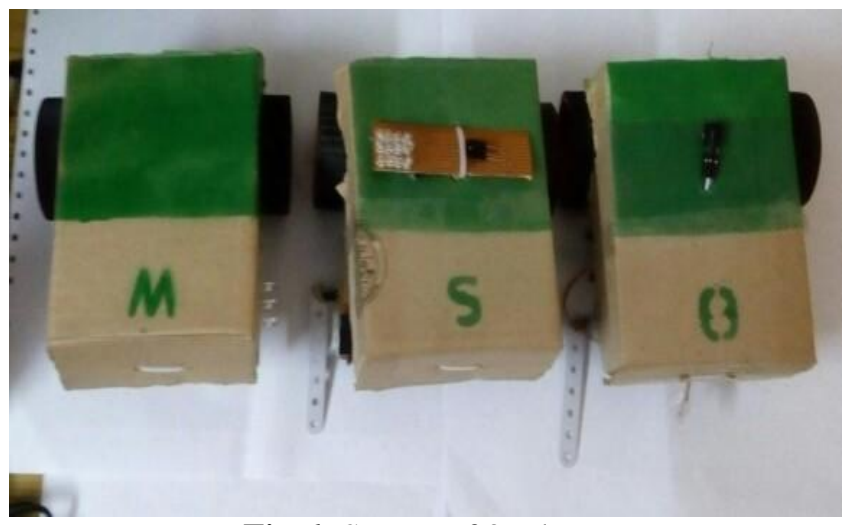

Fig-6: Swarm of 3 robots.

\section{CONCLUSION}

Adaptation of this approach ensures miniaturization and cost effectiveness. The system does not require human supervision. Many tasks can be accomplished by the adaptation of this system. This system will be of great use during natural disasters in removing the debris. Due to smaller size of the ground robots they will be able to sneak into small and confined spaces. This feature will be helpful in identifying humans who are stuck in between debris, if the ground robots are equipped with infrared cameras. As a result of miniaturization, the maneuverability and swiftness certainly increases. The drone once equipped with auto flight feature can make the system more reliable and precise. All the elements of the system are versatile. The drone as an individual element can be used for surveillance. The system accomplishes the task with great precision and ease.

\section{REFERENCES}

[1].A.P.Engelbrecht, Fundamentals of Computational Swarm Intelligence, John Wiley \& Sons,2006.

[2].E.Bonabeau, M.Dorigo and G.Theraulaz,Swarm Intelligence: From Natural to Artificial Systems, Oxford University Press, Inc., New York, NY, 1999.

[3]Y.Meng, O.Kazeem and J.C. Muller, A Hybrid ACO/PSO Control Algorithm for Distributed Swarm Robots, Proceedings of 2007 IEEE Swarm Intelligence Symposium (SIS 2007), pp. 273-280, April 2007.

[4]. J. Kennedy and R.C. Eberhart, Particle Swarm Optimization, Proceedings of IEEE International 
Conference on Neural Networks Vol. IV, pp. 1942-1948.

IEEE service center,Piscataway, NJ, 1995.

\section{BIOGRAPHIES}

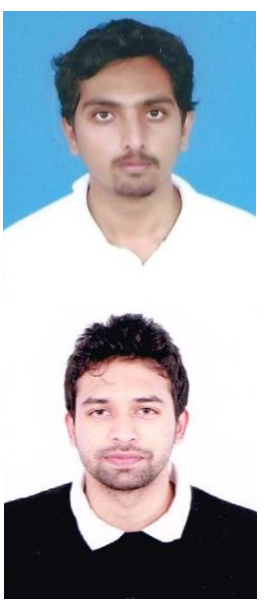

RAJATH U JOSHI

BE (Electronics)

Dayananda Sagar College of

Engineering

Email: rajathjo@gmail.com

\section{SHASHANK T S}

BE (Electronics)

Dayananda Sagar College of

Engineering

Email: shashankts@india.com 